\author{
REVISTA ECONOMÍA \\ Vol. 73, N. ${ }^{\circ}$ II7 (mayo 202I), 29-44 \\ ISSN-i I390-6380, ISSN-e 2697-3332 | https://doi.org/I0.29i66/economia.v73iII7.2955

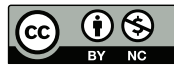

\title{
PRECIOS DE TRANSFERENCIA EN EL COMERCIO EXTERIOR DEL COMPLEJO SOJERO ARGENTINO (2004-2020)
}

\author{
TRANSFER PRICING IN FOREIGN TRADE OF THE ARGENTINE \\ SOYBEAN COMPLEX (2004-2020) \\ GUSTAVO GARCÍA, ${ }^{\mathrm{I}}$ ALEJANDRO GAGGERO ${ }^{2}$ \\ ${ }^{1}$ Universidad Nacional de Rosario, ${ }^{2}$ Universidad de Buenos Aires (Argentina)
}

Recepción manuscrito: 18 de febrero de 2020

Aceptación versión final: 05 de mayo de 2021

\begin{abstract}
RESUMEN El presente trabajo analiza la subfacturación (sobrefacturación) de exportaciones (importaciones) por parte de las empresas del complejo sojero en Argentina durante el período 2004-2020. El artículo sostiene que los mayores desvíos en los precios de transferencia se produjeron en dos períodos específicos: por un lado, en momentos de liberalización en la comercialización, lo que les otorgó a las empresas una mayor libertad de acción; y por el otro, en el contexto de incrementos en los precios internacionales, lo cual les permitió retrasar el aumento del precio pactado con su contraparte relacionada a fin de prolongar la subfacturación.
\end{abstract}

palabras Claves Precios de transferencia en el comercio exterior, complejo sojero, Argentina.

ABSTRACT This paper aims to analyze and account the under-invoicing (over-invoicing) of exports (imports) by the soy grinding complex companies in Argentina during the period 20042020. The article claims that the greatest deviations in transfer prices occurred in two specific periods: on the one hand, in moments of liberalization in commercialization, which gave companies higher freedom of action; and on the other, in the context of increases in international prices, which allowed them to delay the increase in the price agreed with their related counterpart in order to prolong under-invoicing.

KEYWORDS Transfer pricing in foreign trade, soy complex, Argentina.

JEL CODES H26, H87, L11, O12, F23.

\section{INTRODUCCIÓN}

La Argentina es un país en desarrollo que posee un serio problema de restricción externa 
debido a la industrialización incompleta que presenta su estructura productiva y a la desindustrialización prematura que esta sufrió durante las últimas décadas (Azpiazu y Schorr, 2010). Este problema se ha visto agravado además por la desregulación del mercado financiero a partir de mediados de la década de 1970, que potenció el fenómeno de la fuga de capitales hacia los países centrales y las llamadas guaridas fiscales. Si bien no toda la fuga es necesariamente ilegal, la evidencia recabada para Latinoamérica muestra que una proporción importante de ella está vinculada a actividades ilícitas o, en el caso de las legales, estas se encuentran asociadas a algún tipo de evasión impositiva (Gaggero et al., 2013). Este flujo de salida puede darse a través de distintas modalidades, y tan solo como ejemplos, pueden mencionarse: la transferencia de divisas a entidades financieras del exterior, la adquisición de activos físicos - como la compra de inmuebles en otros países - o las maniobras vinculadas al comercio exterior que se analizarán en este artículo.

La escasez crónica de divisas en Argentina ha reforzado el poder de los empresarios exportadores. En el caso del sector oleaginoso, un número acotado de firmas concentran el $28 \%$ de las exportaciones totales del país ${ }^{1}$ y, por ende, aportan el grueso de las divisas comerciales netas. Diversas investigaciones han señalado que en las últimas décadas las firmas del sector han avanzado en la internacionalización de su estructura empresarial a través, por ejemplo, de la creación de filiales en países de baja tributación, lo cual les ha permitido llevar adelante la manipulación de precios de transferencia.

Aunque en los últimos años se ha potenciado la producción académica que analiza los flujos de salida de capitales, la mayoría de los trabajos se han centrado en un análisis de la evolución del proceso de fuga de capitales y sus consecuencias macroeconómicas (Basualdo et al., 2017; Gaggero et al., 2007, 2013; Kulfas, 2005) y, en menor medida, en los vínculos entre las grandes empresas y las filiales en guaridas fiscales (Gaggero, 2015; García Zanotti, 2020). Cabe señalar que a excepción del trabajo de Burgos y Grondona (2015), ${ }^{2}$ no existen análisis sobre la manipulación de los precios de transferencia de las grandes aceiteras en Argentina.

En relación a esta vacancia, el presente artículo se propone analizar la subfacturación (sobrefacturación) de exportaciones (importaciones) por parte de las empresas del complejo de molienda de soja en Argentina durante el período 2004-2020. Para ello se utilizaron como fuentes de información tanto los documentos contables de cada una de las grandes firmas del sector, así como a datos referidos a las transacciones en el comercio exterior efectuadas por estas compañías en el período mencionado. Además, se tuvieron en cuenta las posibles sobrefacturaciones de las importaciones de porotos de soja, las cuales fueron permitidas recientemente a partir de la liberalización comercial durante el gobierno de Cambiemos (2015-2019).

El trabajo se estructura de la siguiente manera. En la primera sección se establecerán ciertas definiciones metodológicas respecto tanto a las guaridas fiscales como a los desvíos en los precios de transferencia y las técnicas utilizadas para su contabilización. En la segunda sección se analizará la internacionalización de las firmas del sector y los desvíos en los precios de transferencia sobre los derivados de soja (harina y aceite) y los porotos de soja. Por último, finalizaremos con una reflexión. 


\section{DEFINICIONES CONCEPTUALES Y METODOLÓGICAS}

Denominamos como guarida fiscal a todo «lugar que procura atraer negocios ofreciendo instalaciones políticamente estables que ayudan a personas o entidades a eludir reglas, leyes y regulaciones en otras jurisdicciones» (Shaxson, 2014, pp. 32-33). Las principales características que poseen dichos espacios extraterritoriales son: bajas tasas impositivas, elevados niveles de secreto bancario, predominio del sector financiero, ausencia de controles de capitales, elevados grados de desregulación en la actividad financiera, falta de transparencia y cooperación en el intercambio de información, entre otras (OCDE, 1996).

Al hablar de empresas offshore, nos remitimos a personas jurídicas constituidas en espacios extraterritoriales. En términos jurídicos, estas empresas se las suele conocer como Special Purpose Entity (SPE) e incluyen a empresas holding, conduit, filiales financieras, sedes regionales, etc. Es decir, son empresas cáscaras que poseen como función mantener participaciones en compañías radicadas en espacios (extra)territoriales, otorgar y recibir préstamos, o detentar la función de oficinas de gestión, ser de intermediarios comerciales, etc. (OCDE, 2008). Las «cáscaras» e intermediarios en términos físicos comprenden únicamente un código postal. En la mayoría de los casos, dichas estructuras se encuentran registradas en un edificio de oficina perteneciente a una corporación dedicada a la actividad de servicios empresariales. En este sentido, al interior de las mismas, no se realiza ningún proceso productivo y, por ende, no poseen trabajadores - en el mejor de los casos, poseen una plantilla muy reducida que se encuentra especializada en tareas de administración-, ni capital fijo.

No existe consenso internacional en lo que respecta a la definición y clasificación de guaridas fiscales, y la lista de países clasificados como tales se encuentra en constante cambio. De hecho, la Organización para la Cooperación y el Desarrollo Económicos (ocDE) ha reducido sustancialmente el número que poseen poca transparencia fiscal y hoy solo clasifica como guarida fiscal a Trinidad y Tobago (Mitchell, 2017). Estudios recientes han mostrado además que el $98 \%$ de los riesgos de fraude fiscal corporativo es responsabilidad de países calificados como «no nocivos» por la oCDE. ${ }^{3}$ En Argentina, la Agencia Federal de Ingresos Públicos (AFIP) considera como jurisdicciones cooperantes a los países donde su respectiva agencia tributaria realiza intercambios de información financiera. ${ }^{4}$ Por la negativa, el resto de países que no integran dicha lista podrían considerarse como guaridas fiscales. Por otro lado, también se entiende como guarida fiscal a toda jurisdicción que posea una tasa tributaria menor que el $60 \%$ de lo que sería efectivamente tributado en Argentina (Ley 27.430 agregados al art. 15 de la Ley de Impuestos a las ganancias).

Desde ya, debemos estar atentos a las diversas jurisdicciones que podrían escapar del radar teniendo en cuenta las particularidades en las prácticas de empresas internacionalizadas en Argentina. En especial, las firmas en Argentina suelen incorporar filiales en países tales como Uruguay y España, dado que estas naciones han liberalizado sus normas durante las últimas décadas para atraer capitales (Deloitte, 2016; Tax Justice Network, 2015). Es decir, dichos países son utilizados como guaridas fiscales al existir leyes laxas respecto a la movilidad de capitales, así como también disponen de bajas o nulas tasas impositivas.

Por otro lado, denominamos como precios de transferencia al valor del pago que se efectúa en una operación entre sociedades vinculadas o relacionadas de un mismo grupo empresarial, 
ya sea por transacciones de bienes, servicios y préstamos, entre otros. Existe un desvío en los precios de transferencia cuando las condiciones de dicha transacción resultan ser diferentes a los que se hubieran pactado entre sociedades independientes - es decir, sin existir una relación de propiedad entre las partes-. En lo que respecta al comercio exterior, las empresas suelen subfacturar (sobrefacturar) las exportaciones (importaciones) para reducir los pagos impositivos y fugar estos fondos hacia filiales relacionadas situadas en países con menores cargas tributarias. Para ello, las empresas internacionalizadas realizan transacciones intrafirma con «cáscaras» de forma de triangular las operaciones a través de estas últimas. Dichas operaciones son realizadas bajo una exhaustiva estrategia de planificación fiscal (tax planning) para minimizar los pagos impositivos.

Las manipulaciones de las condiciones de mercado en las operaciones entre partes relacionadas poseen una concreta incidencia en el pago de impuestos tales como el IVA, el impuesto a las ganancias, las tarifas aduaneras, etc. Dichas operaciones opacas pueden realizarse debido a la existencia de una infraestructura que incluye desde intermediarios comerciales y financieros, así como «facilitadores» (asesores, estudios de abogados y contables, etc.). ${ }^{5}$

La OCDE a través de sus guías de precios de transferencia estipulan y promueven el principio de arm's length, el cual es aceptado tanto entre las agencias tributarias como por parte de los contribuyentes. Este principio establece que las condiciones de mercado de las operaciones pactadas entre partes relacionadas deberían ser equivalentes a aquéllas efectuadas entre empresas no relacionadas. Dichas condiciones de mercado refieren a: el tipo y características del producto, el espacio-tiempo en el cual son realizadas las transacciones, la cantidad del bien operado en la transacción, nivel de oferta y demanda, alcance de la competencia, regulaciones públicas, acondicionamiento (e.g., fletes), unidad de cuenta de la transacción, etc. Por ello, el principio tributario estipula que: Cuando se establecen condiciones comerciales o financieras entre dos empresas asociadas que difieren de las que se realizarían entre empresas independientes, entonces cualquier beneficio que, de no ser por esas condiciones, se haya acumulado en una de ellas, pueden incluirse en las ganancias de esa empresa y gravarse en consecuencia (OCDE, 2017, p. 35). ${ }^{6}$

Existen diversas metodologías para procesar los datos y permitirnos obtener un cálculo sobre el desvío de los precios de mercados fruto al comercio intrafirma, aunque se distinguen cinco principales: 1. precios comparables entre partes independientes (CUP por sus siglas en inglés); 2. precios de reventa entre partes independientes; 3. costo más beneficios; 4. división de ganancias; 5. margen neto de la transacción. Según la guía de precios de transferencia de la OCDE (2017), debe priorizarse el método CUP y solo en caso de ser imposible su cálculo deberá buscarse alternativas metodológicas. La legislación argentina también privilegia la utilización de esta metodología (Decreto Reglamentario, — de ahora en más DR—1344/98 y art. 18 DR 1170/18).

El método cup compara el precio establecido entre partes relacionadas en relación al precio operado en circunstancias similares en transacciones entre partes no relacionadas. En caso de existir diferencias entre ambos precios, estaríamos en la presencia de precios anormales, y por ende, no se cumplirá el principio arm's length. Cabe destacar que tanto la guía de precios de transferencia de la OCDE (2017, párrafo 2.18) como las agencias tributarias de países 
latinoamericanos utilizan el método cup cuando se trata de sectores vinculados a la comercialización de commodities. En este caso el precio de mercado o la cotización comprendería una referencia que nos sirve como comparable respecto a los precios pactados entre partes relacionadas (OCDE, 2017, párrafo 2.19). El método establece como precio de referencia al respectivo precio de cotización internacional del commodity. El desvío entre los precios pactados entre partes relacionadas y el precio de cotización diario constituye la evidencia de subfacturación (sobrefacturación).

Las dificultades a la hora del cálculo de la ganancia producida por parte de la agencia tributaria argentina consisten en las numerosas relaciones intrafirma con filiales en guaridas fiscales. En relación a este punto, existen requisitos que deben ser cumplidos antes de aplicar el presente método. Como menciona la Ley 25.784, este se aplicará: cuando se trate de exportaciones realizadas a sujetos vinculados, que tengan por objeto cereales, oleaginosas, demás productos de la tierra, hidrocarburos y sus derivados, y, en general, bienes con cotización conocida en mercados transparentes, en las que intervenga un intermediario internacional que no sea el destinatario efectivo de la mercadería, se considerará como mejor método a fin de determinar la renta de fuente argentina de la exportación, el valor de cotización del bien en el mercado transparente del día de la carga de la mercadería - cualquiera sea el medio de transporte-, sin considerar el precio al que hubiera sido pactado con el intermediario internacional (art. 2, Ley 25.784).

Desde ya, en caso que el precio internacional «fuera mayor al precio de cotización vigente a la fecha mencionada, se tomará el primero de ellos para valuar la operación». El precio de cotización relevante para ser comparado como precio de mercado será el «correspondiente al día de la carga de la mercadería, el vigente al día en que finaliza dicha carga» (Decreto 916/2004). La aplicación podrá ser llevada a cabo en caso que las exportaciones sean dirigidas a sujetos relacionados o vinculados, siendo la actividad principal de estos últimos la de meros intermediarios de las mismas. Sin embargo, la metodología será descartada en caso que se demuestre sustancia económica por parte del intermediario. En este caso la ley entiende que dos empresas son partes relacionadas siempre y cuando: estén sujetos de manera directa o indirecta a la dirección o control de las mismas personas físicas o jurídicas o éstas, sea por su participación en el capital, su grado de acreencias, sus influencias funcionales o de cualquier otra índole, contractuales o no, tengan poder de decisión para orientar o definir la o las actividades de las mencionadas sociedades, establecimientos u otro tipo de entidades (Ley 27.430, art. 15). Cabe destacar que son muy pocos los casos juzgados y con fallo condenatorio sobre manipulación de los precios de transferencia. Según Grondona y Knobel (2017) esta baja efectividad se debe, en parte, a la dificultad de los organismos estatales para adaptarse a los periódicos cambios regulatorios que se produjeron en los últimos años.

\section{GRANDES EMPRESAS DEL SECTOR EN ARGENTINA Y SU GRADO DE INTERNACIONALIZACIÓN}

El sector oleaginoso argentino se encuentra concentrado en un puñado de empresas. En el caso de la molienda y exportación de los derivados de la soja (harina y aceites en bruto), unas ocho firmas - se trata de Bunge, Cargill, Dreyfus, Cofco, Oleaginosa Moreno Hnos, Aceitera 
General Deheza, Molinos Agro y Vicentin - abarcan el 94\% del mercado (Pérez Barreda et al., 2013). Dentro de ellas se encuentran grandes traders internacionales como Bunge, Cargill, Dreyfus (LDC), Nidera-Noble (Cofco), y Glencore (por medio de Oleaginosa Moreno Hnos.), las cuales conviven con grandes grupos económicos nacionales, como son los casos de Vicentin, Aceitera General Deheza (AGD) y Molinos Agro.?

Tanto los traders internacionales como los grupos económicos desplegaron estrategias de internacionalización, involucrando en su estructura de propiedad a sociedades en guaridas fiscales. Particularmente Dreyfus, Moreno y Nidera se vincularon con estructuras radicadas en Suiza. La firma Noble, del conglomerado Cofco, se mantuvo bajo el control de una estructura en las Islas Vírgenes Británicas, y Bunge de una estructura en las Islas Bermuda. Mientras tanto, el control de Cargill fue ejercido por una estructura en Canadá. En tanto, los grupos económicos locales no poseen un comportamiento diferente al de los traders internacionales. Por un lado, aunque si bien el grupo Vicentin se encuentra controlado por las diversas familias accionistas, éste posee un holding paralelo radicado en Uruguay denominado Vicentin Family Group. Por último, Molinos Agro detenta como controlante a una cáscara en el Estado de Delaware (EE. Uu.).

En segundo lugar, según consta en sus respectivos balances contables, todas las empresas comercian a través de firmas relacionadas en espacios extraterritoriales. Usualmente, las firmas compradoras de los derivados de soja comprenden a cáscaras sin ninguna actividad en la economía «real» y éstas se radican en países de baja tributación y, como tales, actúan como meros intermediarios. En este caso, tanto Bunge, Cargill como Vicentin mantuvieron como principales compradores a firmas relacionadas en Uruguay; mientras que Dreyfus como Noble detentaron como clientes a empresas en Suiza; Oleaginosa Moreno hizo lo propio con una sociedad relacionada en Países Bajos, y, finalmente, Molinos Agro con una filial en Chile. Estos compradores poseen ventajas tributarias por radicarse en espacios extraterritoriales y, de esta forma, ante prácticas de desvíos en el precio pactado, éstos podrán acumular ganancias sin tener la obligación de tributar por ellas. Dichos intermediarios serán los encargados de acumular las diferencias entre la facturación al precio de mercado y la subfacturación del commodity.

Respecto a las importaciones, nuevamente el comercio intrafirma resulta en una característica a destacar. Particularmente, la soja proveniente de Paraguay suele ser exportada por firmas pertenecientes a los mismos traders internacionales o a los grupos locales. En todos los casos, se trata de una empresa intermediaria radicada en Paraguay que realiza la tarea de exportación hacia la Argentina. Al hablar de intermediarios se trata de filiales que no cuentan con capital fijo y no poseen trabajadores a su cargo. Por ejemplo, Vicentin opera bajo una subsidiaria denominada Vicentin Paraguay; Bunge por medio de Bunge Paraguay s. A.; Cargill a través de Cargill Agropecuaria SAIC; y Nidera como Dreyfus por medio de Nidera Paraguay s. A. y LDC Paraguay s. A., respectivamente.

En suma, las firmas del sector poseen un grado elevado de internacionalización que les permite realizar prácticas nocivas a través del comercio intrafirma. Cabe destacar que estas compañías mantienen o mantuvieron procesos judiciales en el tribunal fiscal a partir de actuaciones de la AFIP debido a dichas prácticas en el desvíos de los precios de transferencia (Grondona y Knobel, 2017). 
Figura 1. Empresas del sector oleaginoso: desvíos acumulados por la subfacturación en la exportación de la harina de soja, millones USD (2004-2020)
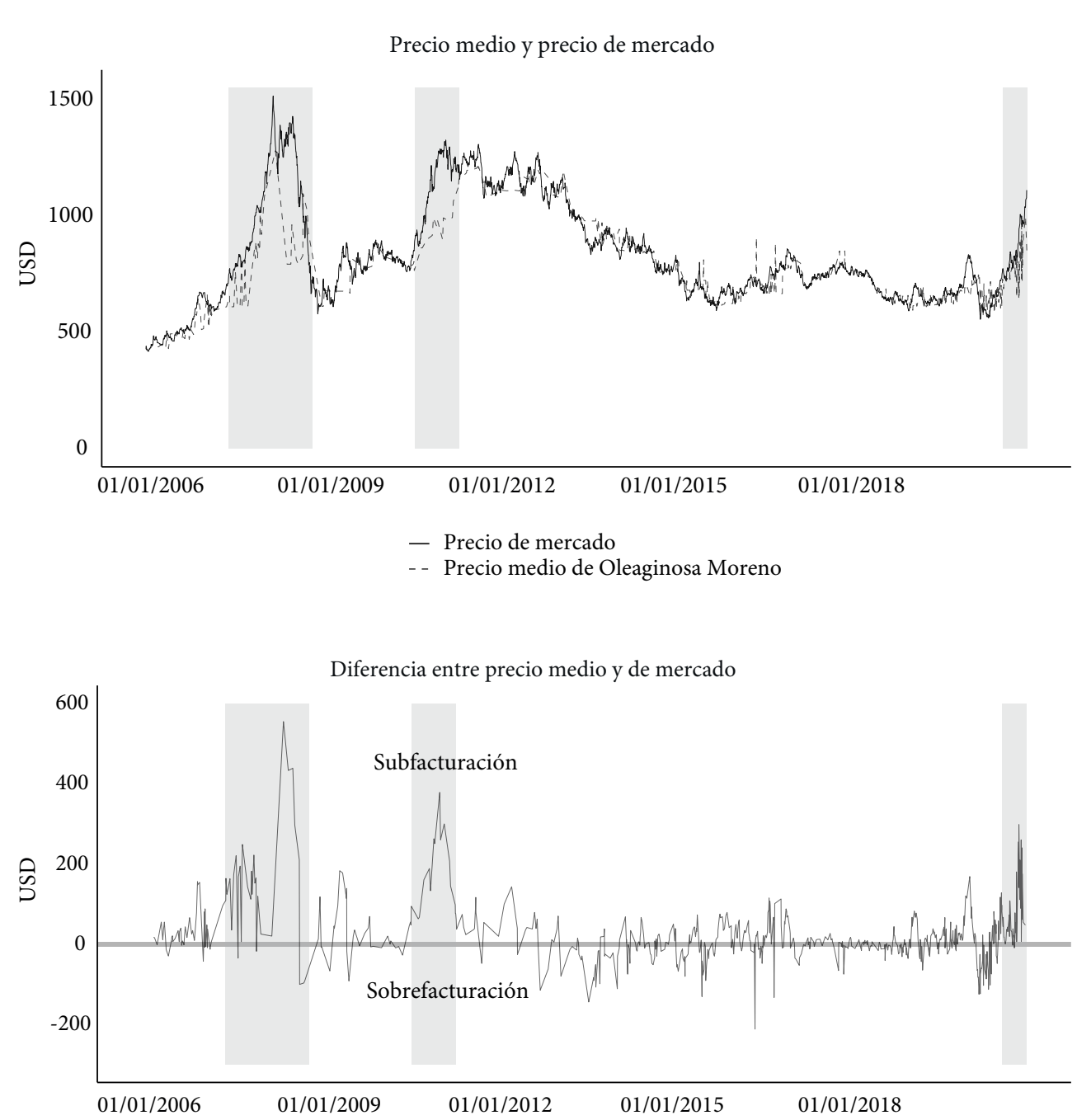

Fuente: Elaboración propia a partir de Softrade y MAGyP.

\section{CONTABILIZACIÓN DE LOS DESVÍOS EN LOS PRECIOS DE TRANSFERENCIA DEL COMPLEJO SOJERO}

En esta subsección analizaremos los desvíos en los precios de transferencia en el comercio intrafirma de las compañías más grandes del sector. Dicho cálculo abarcó unas 104.245 transacciones de comercio exterior correspondientes a la exportación de los derivados de soja y a la importación de porotos durante el período 2004-2020. Para realizar el cálculo de los precios de transferencia, se aplicó el método Cup. Se utilizó como indicador del desvío a la respectiva diferencia entre los precios unitarios de los bienes exportados (pactados con los intermediarios), 
y los precios ғов oficiales del Ministerio de Agricultura, Ganadería y Pesca (MAGyP) en el caso de los derivados de la soja. Mientras tanto se utilizó el precio de mercado de Chicago para la importación del poroto de soja — por ser el mercado de referencia más importante a nivel global para este producto-.

Denominaremos como desvíos acumulados a la sumatoria de dichas diferencias multiplicadas por la cantidad comercializada del respectivo commodity en una unidad de tiempo determinada. Cabe destacar que los precios de exportación oficiales del MAGyP suelen ser utilizados para el cálculo de precios de transferencia por parte del AfIP. Para ello, se tomaron aquellos desvíos que significaron una subfacturación en el caso de la exportación de los derivados de la soja, mientras se aplicó lo propio a la sobrefacturación de las importaciones en el caso del poroto de soja.

\section{EXPORTACIÓN DE DERIVADOS DE SOJA}

Como resultado de la aplicación de la metodología antes mencionada se pudo constatar que estas firmas subfacturaron exportaciones en aquellos momentos de gran variabilidad del precio internacional. Es así que, en los contextos de ascenso del mismo, las compañías exportadoras aumentaron más lentamente sus precios de comercialización, con el objetivo de acumular períodos relativamente largos de subfacturación. En cambio, durante los períodos de baja en el precio internacional, las empresas se acoplaron rápidamente al precio internacional de forma de evitar la sobrefacturación de la exportación del commodity. Es por ello que los períodos de subfacturación resultaron ser más duraderos que aquéllos de sobrefacturación. A lo largo de los años, se evidenciaron grandes diferencias entre el precio de mercado y el precio medio de comercialización pactado con el intermediario, lo cual nos permite verificar la intensidad del fenómeno de subfacturación. En cambio, no ocurrió lo contrario durante los períodos de baja en el precio internacional, siendo estos los que posibilitarían la sobrefacturación.

En relación con este punto, para entender más de cerca el funcionamiento de estos desvíos entre el precio pactado con el intermediario y precio internacional, hemos tomado como caso práctico a la empresa Oleaginosa Moreno Hnos. (Glencore). Para ello, se construyó una variable que comprende el precio medio unitario diario correspondiente a aquellas transacciones con el intermediario (ver Figuras 1 y 2 del anexo digital). A esta última se la comparó con el precio internacional. Los precios pactados con la empresa relacionada —en los gráficos a través del precio medio de Oleaginosa Moreno Hnos. - suelen seguir de cerca al internacional. Sin embargo, durante los períodos de ascenso de este último, el precio pactado con la empresa relacionada quedó rezagado de tal evolución. En cambio, cuando el precio internacional decayó, el precio pactado con la firma relacionada se acopló rápidamente. Por esta razón, la diferencia entre dichas variables mostró grandes saltos en el campo de la subfacturación, y no así en el de la sobrefacturación.

En este sentido, respecto a la comercialización de harina de soja, durante el nuevo milenio se produjeron cuatro períodos de ascenso en los precios internacionales que les permitieron a las firmas acumular desvíos en los precios de transferencia: 2007-2008, 2012, 2016 y 2020 (ver Figura 1). Por esta razón, podemos visualizar estos períodos de ascenso en su contabilización debido a la acumulación de estos desvíos por subfacturación. El desvío por subfacturación 
Figura 2. Empresas del sector oleaginoso: desvíos acumulados por la subfacturación en la exportación del aceite de soja, millones USD (2004-2020)

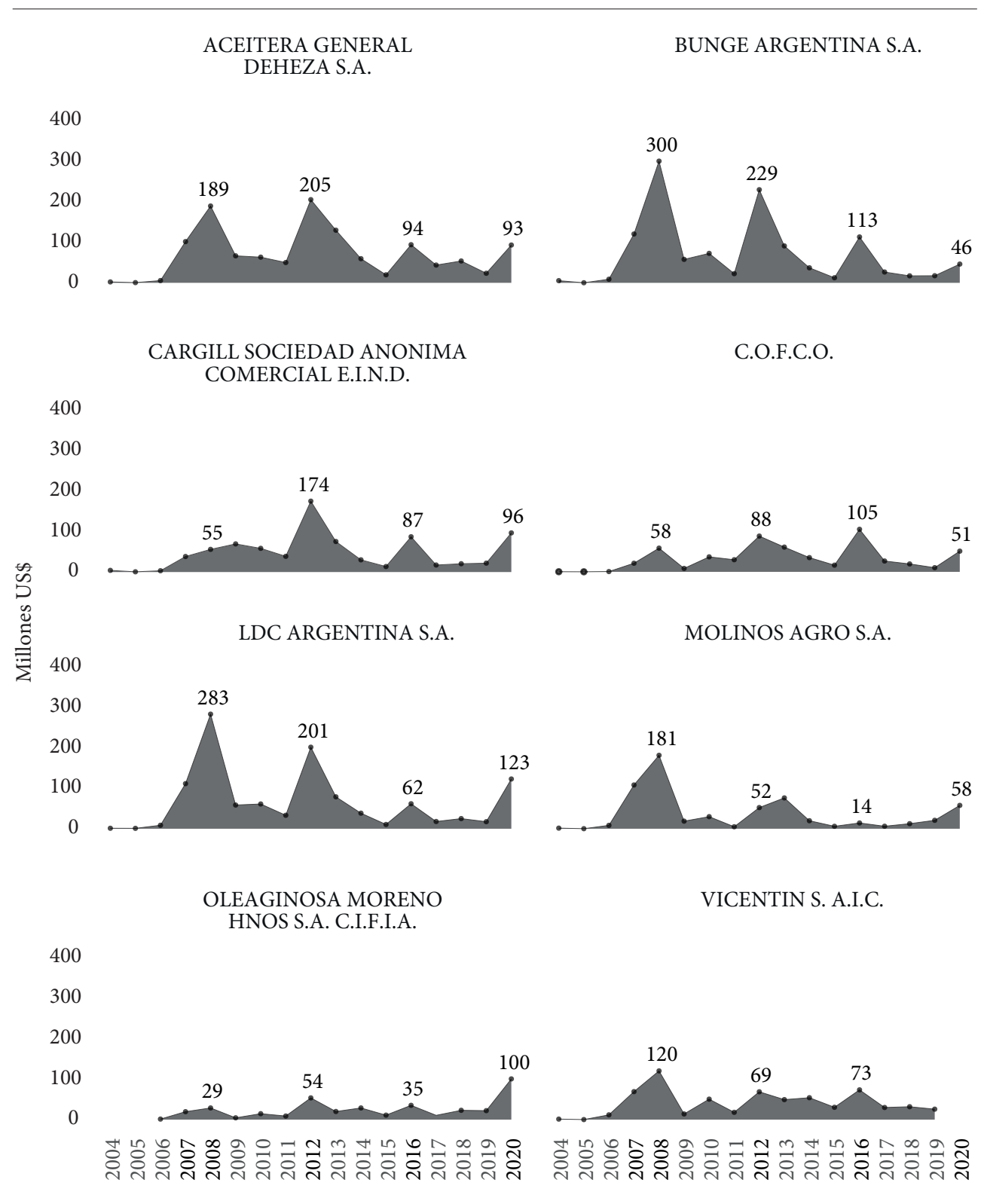

Fuente: Elaboración propia a partir de Softrade y MAGyP.

del año 2008 fue especialmente elevado dado el contexto de crisis internacional e incremento de los precios internacionales. De esta forma, los traders internacionales aprovecharon la ocasión para realizar tales prácticas de forma de proveer liquidez a sus casas matrices. Las firmas 
que más se destacaron fueron las compañías extranjeras como Cargill, Bunge y Dreyfus. Así también dentro de los grupos económicos nacionales sobresalieron tanto AGD como Vicentin.

En el caso de la comercialización de aceite de soja, los mayores desvíos en los precios de transferencia se produjeron en los años 2007-2008, 2010, 2016 y 2020 (ver Figura 2). Estos saltos en los precios de transferencia acumulados coincidieron con aumentos del precio internacional del respectivo commodity. Nuevamente se repiten los mismos actores con los mayores desvíos acumulados, entre los que se encuentran: Cargill, Bunge y Dreyfus en el caso de los traders internacionales, y AGD y Vicentin para los grupos nacionales.

En ambos derivados de la soja, debería destacarse el aumento producido en los desvíos acumulados de la firma Oleaginosa Moreno Hnos. para el año 2020. Esto se debe al aumento en su producción gracias al concurso de quiebra de Vicentin, ya que la firma internacional acaparó el mercado vacante dejado por aquel tras dicho proceso. En su totalidad, para el período bajo estudio (2004-2020), el monto de subfacturación del sector de ambos derivados de la soja alcanzó unos USD 11.000 millones y representó en promedio el 5\% de las exportaciones. Aunque, como mencionamos, se evidenciaron años en los cuales estas diferencias resultaron ser mayores. Por esta razón, el ratio ascendió al 12\% para aquellos años de aumento de los desvíos, y del $3 \%$ en el resto del período.

Finalizando, se torna útil estudiar los destinos que muestran los mayores desvíos entre el precio pactado y el precio de mercado. Países como España y Países Bajos mostraron los montos acumulados más importantes de precios de transferencia. Sin embargo, estos países no constituyen los destinos más representativos en términos de los volúmenes comercializados, siendo el sudeste asiático quien acapara dicho destino.

Particularmente, Países Bajos funciona como una guarida fiscal en la cual se localizan muchas de las Special Purpose Entities (SPE). Éstas funcionan como intermediarias de los préstamos intrafirma y el comercio exterior. Aunque se desconoce si la mercadería tuvo tal destino o si dicho país responde a la radicación de la intermediaria en lo que respecta a la facturación de la exportación. Muchos de los grandes traders comercializan con SPE en Países Bajos. Es el caso de Oleaginosa Moreno con Glencore Grain B. v., filial radicada en dicho país; Dreyfus con Nethgrain; o Nidera Argentina con Nidera B. v. Además, cabe destacar que el puerto de Róterdam (Países Bajos) suele ser utilizado para realizar reexportaciones.

En el mismo sentido que en el punto anterior, España es utilizada por empresas internacionalizadas para desplazar sus ganancias hacia destinos offshore. Dentro de los casos estudiados, Vicentin saIc detenta una filial en dicho país que funciona como intermediario de los commodities exportados. En definitiva, las prácticas nocivas de subfacturación se sustentaron en los mecanismos de internacionalización en un sector que presenta una elevada concentración. Estos desvíos presentaron una importancia relativa durante el período bajo estudio en general como en los años de incremento del precio del commodity en particular (ver Figuras 3 y 4).

\section{IMPORTACIÓN DE POROTOS DE SOJA}

En la presente subsección, explicaremos el régimen de importaciones de soja en Argentina y las prácticas nocivas que poseen las empresas al realizar precios de transferencia. En primer lugar, cabe destacar que las compras de soja desde el exterior se reglamentaron bajo el carácter 
Figura 3. Precio de mercado del aceite de soja, precio medio de Oleaginosa Moreno Hnos. pactado con el intermediario y la diferencia entre estos valores, en USD (2006-2020)
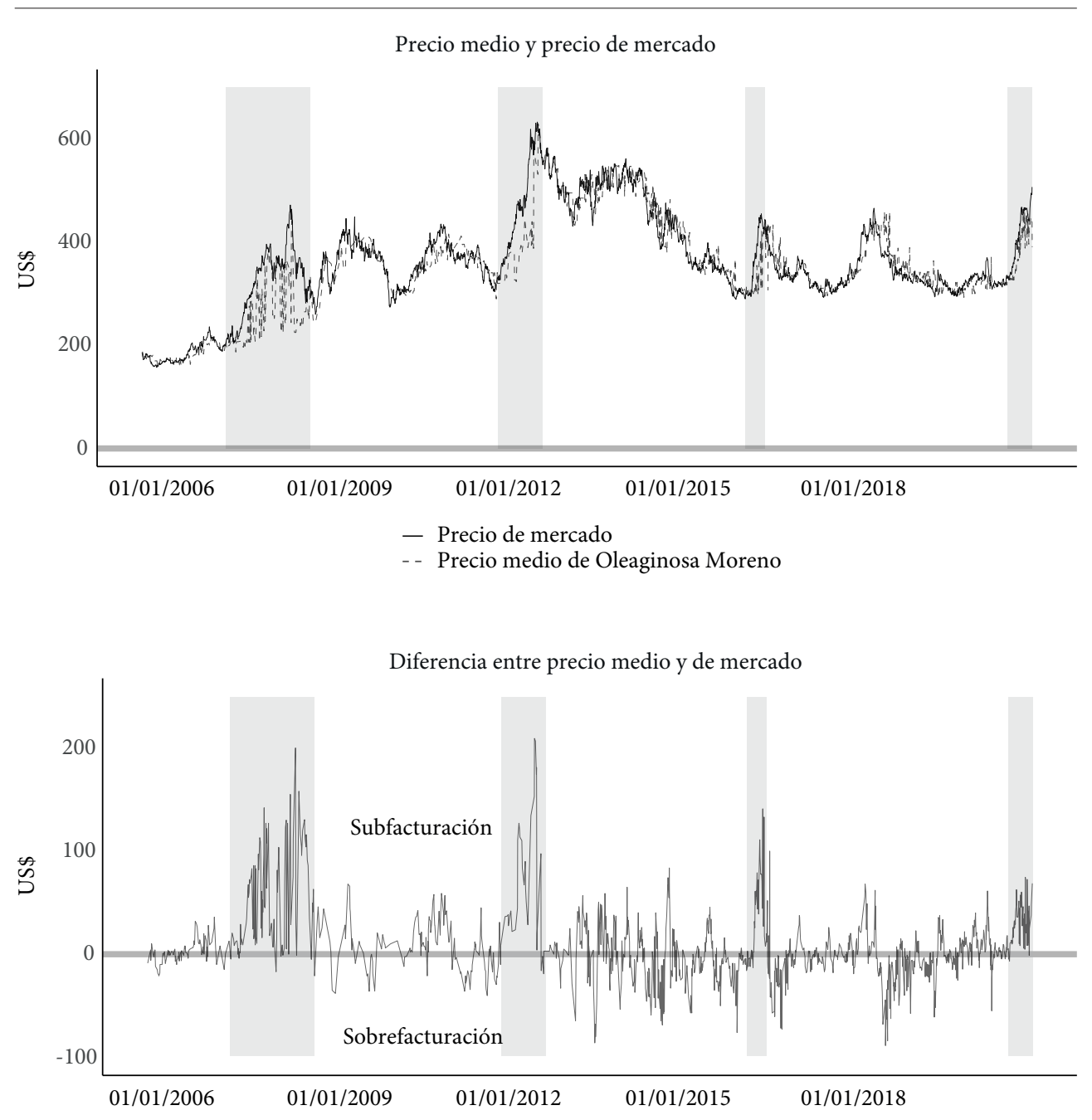

Fuente: Elaboración propia a partir de Softrade y MAGyP.

de importaciones temporarias. Estas comprenden a «[...] mercaderías destinadas a recibir un perfeccionamiento industrial, con la obligación de exportarlas para consumo a otros países bajo las nuevas formas resultantes, dentro del plazo autorizado» (Decreto 1330/2004). Estas importaciones consisten en un régimen aduanero y tributario que permite importar ciertos productos sin aranceles ni tributos con el fin de agregarles valor en el país para luego exportarlos en un plazo determinado. En la Argentina, las importaciones temporarias fueron reglamentadas por el Decreto 1330/04. 
Durante los gobiernos kirchneristas (2003-2015), las importaciones de soja se desarrollaron especialmente en los primeros años de este período, y con mayor énfasis en los años 20072009. Posteriormente este régimen fue limitado y en los hechos quedó en desuso durante los años 2010-2015. Sin embargo, el gobierno de la Alianza Cambiemos modificó dicho régimen de importación por medio de los decretos 523/2017 y 854/2018 (entre otros), los cuales buscaron su flexibilización. En la mayoría de las grandes empresas del sector, las importaciones comenzaron a crecer a partir de 2016. No obstante, no fue sino hasta el año 2018 que las mismas se incrementaron con fuerza debido a la sequía de la campaña 2017/2018. La procedencia de las mismas comprende a Paraguay, Estados Unidos y Brasil.

A partir de la última flexibilización importadora, el mercado quedó aún más concentrado en comparación con el de exportación de derivados de soja. De esta forma, Vicentin saIC se constituyó en el mayor importador de soja y sobrepasó a su competencia con facilidad durante los años 2018-2019. Para el año 2019, Vicentin SAIC detentaba el 33\% de las importaciones de porotos de soja. Tras el cambio de gobierno a finales de 2019, el régimen de importaciones temporarias siguió vigente. Sin embargo, el concurso de quiebra de la empresa Vicentin alteró el esquema de actores beneficiados de dicho régimen. De esta forma, para el año 2020, Oleaginosa Moreno, empresa aliada de Vicentin, ${ }^{8}$ acaparó la participación de esta última, dado que la misma no pudo operar en el mercado. Como consecuencia, las exportaciones del trader internacional crecieron fuertemente en el año 2020 y, como resultado, éste explicó el 46\% de las importaciones de porotos de soja de la Argentina.

Este cambio de beneficiarios del régimen de importaciones se debe al propio comercio intrafirma desde Paraguay. Cabe destacar que Vicentin Paraguay s. A. declaró no haber realizado operaciones de exportaciones hacia la Argentina en el año 2020, debido al concurso de acreedores. Sin embargo, emergió un nuevo actor en las exportaciones paraguayas de porotos de soja, el cual no poseía ninguna participación en años anteriores. Se trata de Glenpar s. A., una filial del conglomerado Glencore que desplazó a Vicentin Paraguay en la participación del mercado de exportación paraguayo. De esta forma, existió una clara colusión entre Vicentin y el conglomerado Glencore para trasladarle su cuota de mercado a este último. Como resultado, Oleaginosa Moreno Hnos. se constituyó para el año 2020 en el mayor exportador del sector de la Argentina gracias al incremento de las importaciones desde Paraguay.

En relación con el punto anterior, cabe destacar que, en términos de facturación, los compradores de la soja paraguaya no se correspondieron con las contrapartes argentinas. En este sentido, existieron otros intermediarios en el mismo comercio intrafirma. Es así, los compradores de la soja paraguaya con destino a la Argentina estuvieron explicados por la sucursal uruguaya de Vicentin en el caso del grupo nacional, como por Glencore Agriculture B. v. (Países Bajos) en el caso de Oleaginosa Moreno Hnos. Es decir, el commodity fue vendido a una filial en una guarida fiscal para luego ser adquirido por la firma en territorio nacional.

Debido a estas compras intrafirma, los montos de las exportaciones de Paraguay a la Argentina no coincidieron con las importaciones de este último país por aquél. En el año 2020, las exportaciones de soja desde Paraguay hacia Argentina ascendieron a unos USD 1588 millones, mientras que las importaciones de Argentina desde Paraguay totalizaron USD 1824 millones. Estas diferencias se producen debido a la respectiva facturación por parte de 
Figura 4. Precio de mercado de la harina de soja, precio medio pactado con el intermediario de Oleaginosa Moreno Hnos. y la diferencia entre estos valores, en USD (2006-2020)

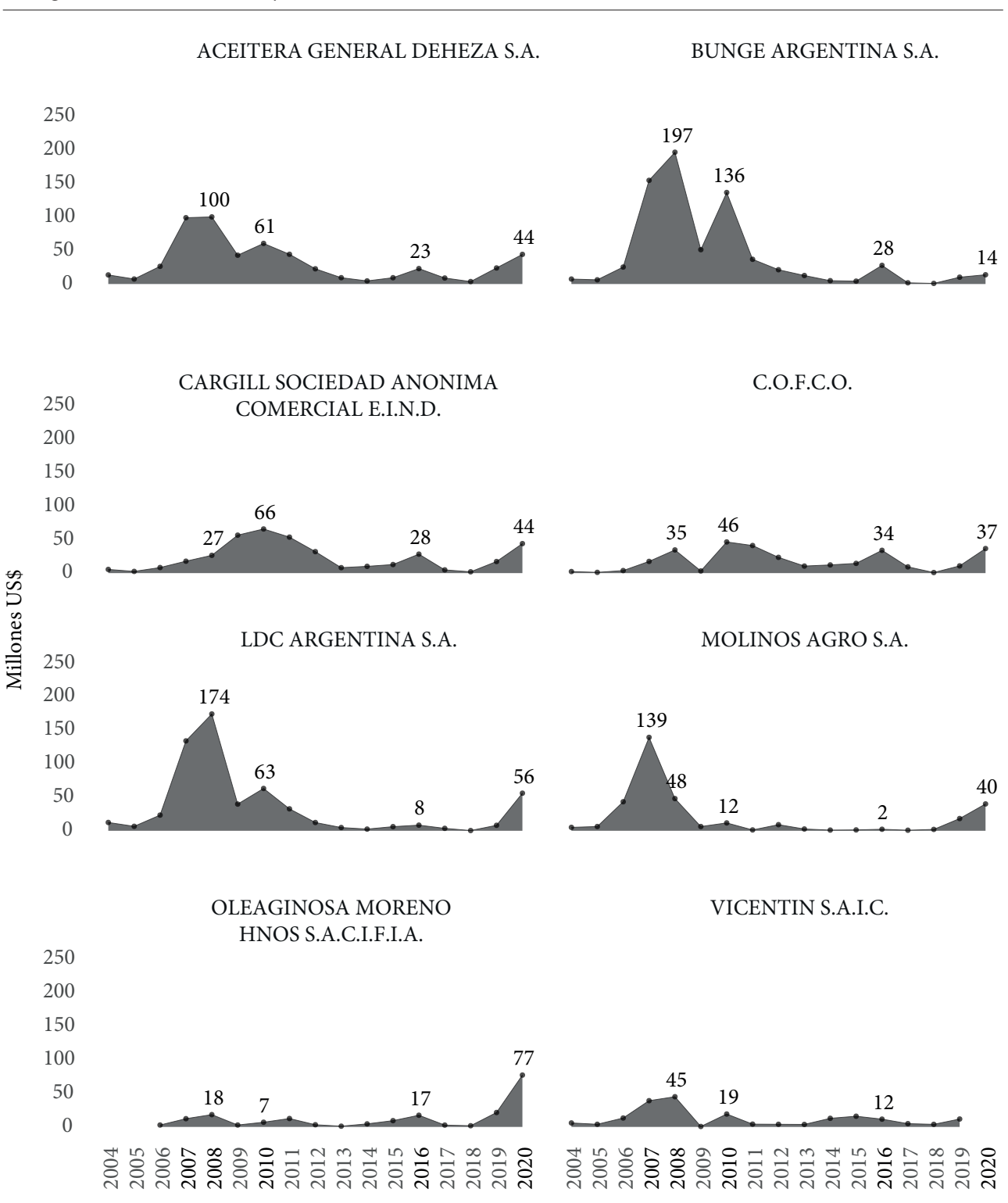

Fuente: Elaboración propia a partir de Softrade y MAGyP.

intermediarios que opacan las transacciones. Por ejemplo, Glenpar declaró haber exportado soja a Países Bajos por unos usD 94 millones en el año 2020, sin embargo, lo más probable es que se trataría de soja que tuvo como destino a la Argentina y por la cual intervino un intermediario de dicho origen. 
Por esta razón, la importación de soja debería entrar dentro de las consideraciones que incluyan prácticas especulativas de desvíos en los precios de transferencia. De igual manera que el punto anterior sobre los derivados de soja, en esta oportunidad se calcularon los desvíos acumulados que comprenden a la sobrefacturación del comercio intrafirma (ver Tabla 1). En el caso de las importaciones, no existieron grandes desvíos en los años 2008-2009, momento en el que se habían realizado las mayores importaciones durante la etapa de los gobiernos kirchneristas. En tanto, en los años más recientes, se produjeron desvíos más significativos, especialmente a partir del año 2018 cuando las importaciones aumentaron por la sequía. En este caso, se destacaron Vicentin, Oleaginosa Moreno Hnos., Cargill y Nidera en tales prácticas. Sin embargo, estos desvíos menguaron significativamente en el año 2019, siendo Oleaginosa Moreno Hnos y Vicentin las únicas en mostrar diferencias que resultaron ser más bien reducidas respecto al año anterior. Mientras tanto para el año 2020, Oleaginosa Moreno Hnos. acaparó gran parte de tales prácticas dada la mayor concentración en el mercado en la importación de soja antes mencionada. Por último, cabe resaltar que el monto de sobrefacturación representó en promedio el $3 \%$ de las importaciones en aquellos años de vigencia del régimen de importaciones.

\section{REFLEXIONES FINALES}

Un puñado de empresas nacionales e internacionales concentra prácticamente la totalidad del mercado de molienda y exportación de soja en la Argentina. Estas empresas poseen un elevado grado de internacionalización que les permite triangular el comercio exterior por medio de firmas relacionadas que actúan como meros intermediarios. Estos últimos están localizados en países de baja tributación, que resultan ser espacios propicios para acumular las diferencias entre el precio de mercado y la subfacturación (sobrefacturación) de las exportaciones (importaciones).

En el período bajo análisis, las mayores diferencias se produjeron en los momentos en los cuales los precios internacionales subieron súbitamente. En dichos contextos, las empresas exportadoras retrasaron el incremento del precio pactado con su contraparte relacionada dado que de esta forma podrían prolongar el período de subfacturación. Entre las firmas que acumularon mayores diferencias se encuentran tanto los traders internacionales como los grupos nacionales. En tanto, el monto de subfacturación representó una parte importante de las exportaciones, especialmente en aquellos contextos propicios.

Por otra parte, el mercado de la importación de soja se presentó aún más concentrado que el de exportación. En este caso, principalmente Vicentin y luego Oleaginosa Moreno acaparan buena parte del mismo durante los últimos años del período bajo estudio, y comercializaron el commodity por medio de intermediarios. Bajo estas condiciones, dichos actores detentaron los principales desvíos entre los precios pactados y los precios de mercado.

En definitiva, estas prácticas resultan ser sumamente nocivas para países que poseen serias restricciones externas como es el caso de la Argentina. Estamos hablando del sector que realiza los mayores aportes netos en términos de divisas. Sin embargo, por este motivo, estas firmas poseen un poder de mercado determinante que las habilita a desplegar tal tipo de comportamiento. 
Tabla 1. Empresas del sector oleaginoso argentino: desvíos acumulados por sobrefacturación de las importaciones del poroto de soja, en millones US\$ (años particulares)

\begin{tabular}{|c|c|c|c|c|c|}
\hline Empresas & 2008 & 2009 & 2018 & 2019 & 2020 \\
\hline Aceitera General Deheza SA & 4 & 1 & 2 & 1 & 3 \\
\hline Bunge Argentina S.A. & 6 & 2 & 9 & 0 & 1 \\
\hline Cargill SAIC & 12 & 12 & 18 & 1 & 8 \\
\hline LDC Argentina S.A. & 4 & 0 & 6 & 0 & 3 \\
\hline Nidera S.A. & 0 & 0 & 16 & 0 & 4 \\
\hline Oleaginosa Moreno Hnos & 0 & 0 & 31 & 10 & 59 \\
\hline Vicentin SAIC & 1 & 0 & 57 & 12 & 0 \\
\hline Total & 27 & 15 & 139 & 24 & 78 \\
\hline
\end{tabular}

Fuente: Elaboración propia a partir de Softrade y Mercado de Chicago. Fuente: Elaboración propia a partir de Softrade y MAGyP.

\section{NOTAS}

1 Datos del INDEC sobre el año 2019.

2 El presente trabajo no pretende innovar respecto a la metodología aplicada por Burgos y Grondona (2015), más bien las diferencias deberían encontrarse en el espacio temporal analizado dado que este último se acota únicamente al período 2010-2013, mientras que en nuestro caso tomamos al período 2004-2020. Además, el presente artículo analiza las dinámicas de sobrefacturación en las importaciones las cuales no se encuentran disponibles en otros cálculos realizados hasta el momento.

3 Ver https://www.taxjustice.net/press/la-clasificacion-de-paraisos-fiscales-muestra-que-los-paises-qu e-establecen-las-normas-fiscales-mundiales-hacen-lo-posible-para-ayudar-a-las-empresas-a-ignoralas/

4 Ver la lista en línea: http://www.afip.gob.ar/jurisdiccionesCooperantes/\\#ver

5 Un conocido ejemplo de «facilitador» es el estudio Mossack Fonseca, expuesto a la opinión pública gracias al trabajo del Consorcio Internacional de Periodistas de Investigación. A partir de una filtración de documentos de este estudio panameño, pudieron identificarse más de 200.000 empresas creadas en guaridas fiscales.

6 Grondona y Knobel (2017) señalan que en Argentina el principio de arm's length comenzó a ser utilizado por las autoridades fiscales a partir de 1976, suplantando al principio de realidad económica.

7 Por grupo económico, se entiende al conjunto de empresas legalmente independientes ligadas por lazos formales de propiedad y ubicadas en distintos sectores de propiedad. Los grupos económicos son organizaciones empresariales que han tenido un rol destacado en la economía mundial durante los últimos cuarenta años. Su papel fue especialmente importante en los países periféricos, donde se expandieron en paralelo a los procesos de industrialización tardía (Amsden, 1989).

8 El conglomerado Glencore y Vicentin comparten participaciones en la empresa Renova. Además, la multinacional es habitual cliente de la firma nacional.

\section{REFERENCIAS}

Amsden, A. (1989) Asia's Next Giant: South Korea and Late Industrialization. Oxford University Press. Azpiazu, D. y Schorr, M. (2010). Hecho en Argentina: industria y economía, 1976-2007. Siglo xxi Editores. Basualdo, E. (ed.), Wainer, A., Barrera, M., Bona, L., González, M., Manzanelli, P. y Wainer, A. (2017). Endeudar y fugar. Un análisis de la historia económica argentina, de Martínez de Hoz a Macri. Estado y Políticas Públicas, 6(10), 231-235. 
Burgos, M. y Grondona, V. (2015). Fuga de capitales vi. Estimación de los precios de transferencias. El caso del complejo sojero. CEFIDAR.

Deloitte. (2016). Taxation and investment in Spain 2016. Deloitte Touche Tohmatsu Limite.

Gaggero, A. (2015). Fuga de capitales vir. los efectos de la internacionalización y extranjerización de los grandes grupos empresarios argentinos. los casos de techint, eurnekian y fortabat. CEFID-AR, Documento de trabajo $n .^{\circ} 74$.

Gaggero, J., Casparrino, C. y Libman, E. (2007). La fuga de capitales: historia, presente y perspectivas.

Gaggero, J., Rua, M. y Gaggero, A. (2013). Fuga de capitales III. Argentina (2002-2012).Magnitudes, evolución, políticas públicas y cuestiones fiscales relevantes. CEFID-AR, Documento de trabajo $n .^{\circ} 52$.

García Zanotti, G. (2020). Contabilización de la fuga de capitales en el complejo aceitero de Argentina y Brasil durante el nuevo milenio (2001-2017). Divulgatio. Perfiles Académicos de Posgrado, 4(12).

Grondona, V. y Knobel, A. (2017). Transfer pricing in Argentina 1932-2015.

Kulfas, M. (2005). Internacionalización financiera y fuga de capitales en América Latina. Flacso.

Mitchell, R. (2017). OECD Lists one tax haven, tax justice network says 'nonsense!' Bloomberg: https:// Www.Bna.Com/Oecd-Lists-One-N73014460947/.

OCDE. (2017). Transfer pricing guidelines for multinational enterprises and tax administrations 2017.

OCDE (1996). OECD Benchmark definition of foreign direct investment, Third edition. OCDE.

OCDE (2008). OECD Benchmark definition of foreign direct investment. Fourth edition. OCDE.

Pérez Barreda, N., Kofman, M. y Abraham, L. (2013). El capital extranjero en la transformación de la industria aceitera argentina de las últimas décadas (1970-2010). Jornadas de Economía Crítica.

Shaxson, N. (2014). Las islas del tesoro. Los paraísos fiscales y los hombres que se robaron el mundo (B. Aires [ed.]). Fondo de Cultura Económica.

Tax Justice Network (2015). Narrative report on Uruguay. Financial Secrecy Index. 